\title{
UNA MEDIDA DE LA HOMONEGATIVIDAD BREVE, UNIDIMENSIONAL, E INDEPENDIENTE DE LOS AFECTOS POSITIVOS Y NEGATIVOS: EVIDENCIAS DE VALIDEZ DE LA ESCALA DE WRENCH EN CHILE
} A BRIEF AND MONO-DIMENSIONAL HOMONEGATIVITY MEASURE, INDEPENDENT
FROM POSITIVE AND NEGATIVE AFFECTS:
EVIDENCE OF WRENCH'S SCALE VALIDITY IN CHILE DOI: 10.22199/S07187475.2016.0001.00001

Recibido: 22 de febrero del 2016 | Aceptado: 29 de marzo del 2016

FELIPE QUEZADA CONCHA 1

(FUNDACIÓN IGUALES, Talca, Chile)

\begin{abstract}
RESUMEN
INTRODUCCIÓN: En Chile existen escasas investigaciones sobre la homonegatividad, constructo que combina cogniciones, afectos y conductas de carácter negativo hacia las personas homosexuales (Hudson \& Ricketts, 1980). OBJETIVOS: Evaluar la fiabilidad y validez de la Escala Breve de Homonegatividad, determinar si existe la suficiente invarianza factorial para realizar comparaciones demográficas y evaluar la influencia del Estado Emocional en la expresión de la homonegatividad. MÉTODO: Aplicación a 303 sujetos (29,4\% hombres, 70,6\% mujeres, media de 23,25 años de edad (DT= 5,5 años) de batería compuesta por la Escala Breve de Homonegatividad (Wrench, 2005), cuestionario de información demográfica y el Inventario Abreviado de Emocionalidad Positiva y Negativa (PANAS short form) (Thompson, 2007) mediante un muestreo por conveniencia tipo "bola de nieve" a través de internet. RESULTADOS: La escala demostró ser unifactorial, ser invariante tomando en cuenta el sexo de los respondientes, pero sólo a un nivel configural-débil, donde los hombres alcanzaron puntuaciones levemente más altas que las mujeres, estando, sin embargo, ambos grupos en un nivel bajo de homonegatividad. CONCLUSIONES: la Escala Breve de Homonegatividad posee propiedades psicométricas que la hacen ser un instrumento adecuado y válido para cuantificar la homonegatividad en Chile, donde el nivel de homonegatividad manifiesto resulta ser independiente del estado emocional del sujeto al momento de responder. El contar con esta herramienta breve de screening permite describir de mejor forma este fenómeno, donde la generación de nuevos estudios en la materia permitirá a futuro su erradicación en la sociedad chilena.
\end{abstract}

PALABRAS CLAVE: Homonegatividad, análisis factorial confirmatorio, invarianza, modelos de ecuaciones estructurales, afecto.

\section{ABSTRACT}

Introduction: Homonegativity -multidimensional construct that consists of negative affective, cognitive and behavioural responses directed towards homosexuals (Hudson \& Rickets, 1980)-, is an emerging research topic in Chile. Objectives: i. To evaluate the reliability and validity of the Homonegativity Short Form. ii. To determine if there is enough factorial invariance to make demographical comparisons. iii. To evaluate the influence of the Emotional State in homonegativity. Method: The Homonegativity Short Form (Wrench, 2005), a questionnaire of demographic information and the Positive and the Negative Affect Schedule (PANAS short form) (Thompson, 2007) were applied to a sample of 303 subjects ( $29,4 \%$ men / 70,6 \% women), with an average age range of 23. To collect data convenience sampling was used through the snowball sampling technique via internet. Results: The scale proved to be unifactorial, and also invariant considering the gender of informants. Although men slightly reached higher scores than women, results overall indicate both groups have a low level of homonegativity. Conclusions: The Homonegativity Short Form is an appropriate and valid instrument to quantify homonegativity in Chile. Based on their answers, the emotional state of informants did not influence the level of homonegativity. This instrument describes homonegativity very well. It is believed new studies on the subject will allow the eradication of homonegativity in Chilean society in the future.

KEY WORDS: Homonegativity, confirmatory factorial analysis, invariance, structural equation models, affect.

1. Psicóloga, afiliado a la Fundación Iguales, Talca, Chile. E-mail: felip.quezada@gmail.com 


\section{INTRODUCCIÓN}

Las actitudes hacia la homosexualidad han sido objeto de investigación en la literatura desde principios de 1980, desarrollando múltiples conceptualizaciones, modelos teóricos e instrumentos para su medida (Grey, Robinson, Coleman \& Bockting, 2013). La mayoría de la investigación en este campo se han realizado bajo el uso del constructo "homofobia", definida como el miedo de las personas heterosexuales a estar en un mismo lugar con gente homosexual, 0 en el caso de un(a) homosexual, autoaversión (Weinberg, 1972). La creación del término fue clave en generar una visión despatologizante de la homosexualidad, que fue respaldada por la Asociación Americana de Psiquiatría en 1973 (Bayer, 1981; Minton, 2002), por la Organización Mundial de la Salud en 1990 (WHO, 2010, 2011) y por la Asociación Americana de Psicología en 1975 (Clarke, Ellis, Peel \& Riggs, 2010). Sin embargo, esta definición ha sido históricamente criticada (Millham, San Miguel \& Kellogg, 1976; Fyfe, 1983; Herek, 1984; Logan, 1996; Adam, 1998; Borillo, 2001; Wrench, 2005; Lottes \& Grollman, 2010; Chamberland \& Lebreton, 2012), debido a que las actitudes hacia las personas gays 0 la interacción social con éstos, no necesariamente se manifiestan con activaciones fisiológicas relacionadas a las fobias (e.g., sudoraciones, palpitaciones, conducta de evitación, asco); más bien al contrario, se trata de reacciones emocionales con carácter funcional. El uso del sufijo fobia supondría una aversión incontrolable, librando de responsabilidades a quien la ejerce y obviando hostilidades menos irracionales (Hudson \& Ricketts, 1980; Bhugra, 1987; Haaga, 1991; Borrillo, 2001; Shields \& Harriman, 1984; Carneiro, 2009; Chamberland \& Lebreton, 2012).

A partir del concepto original de homofobia han surgido múltiples acepciones: homofobia como un rasgo de la personalidad (Smith, 1971; Fyfe, 1983;
Parrot, Adams, Zeichner, 2002), como un sentimiento (Bell, 1989), como un componente basado en los valores (O'Donohue \& Caselles, 1993), como una conducta (Gray, Russell \& Bockley, 1991), como un miedo irracional (MacDonald, Huggins, Young \& Swanson, 1973), como un proceso cultural (Reiter, 1991) o como una serie de conductas discriminatorias (Baral, Sifakis, Cleghorn \& Beyrer, 2007; Cáceres, Pecheny, Frasca, Ríos, Pocahy, 2008; Harding, 2010). Algunos autores proponen que lo más acertado sería el uso de homonegatividad u homonegativismo (término utilizado en el presente estudio). La homonegatividad combina cogniciones, afectos y conductas de carácter negativo hacia las personas homosexuales o quienes socialmente parecen serlo, contemplando creencias prejuiciosas, reacciones emocionales y conductas de estigmatización (Hudson \& Ricketts, 1980; Roderick, McCammon, Long \& Allred, 1998; Currie, Cunningham \& Findlay, 2004, Morrison \& Morrison, 2011). El uso de homonegatividad, parece más acorde a la realidad actual y evitaría la relación con una fobia clínica (Wrench, 2005), aunque el uso popular y académico del término homofobia aun persiste (Bhugra, 1987; Dermer, Smith \& Barto, 2010).

Los modelos teóricos de la homonegatividad han planteado diversas estructuras que están reflejadas en los instrumentos de medida disponibles actualmente. Las dimensiones más frecuentemente propuestas tienen que ver con actitudes (a) hacia las propias personas homosexuales (Kite \& Withley (1996), (b) hacia la conducta homosexual (Kite \& Withley, 1996), (c) hacia las relaciones de pareja homosexuales (Lewis, 2003), (d) hacia los derechos laborales (Lewis, 2003), (e) ante la igualdad de derechos y libertades civiles de los homosexuales (Kite \& Withley, 1996; Yang, 1997; Davies, 2004; Lewis, 2003), (f) hacia el matrimonio y la adopción homoparental (Yang, 1997), (g) reacciones emocionales $y$ afectivas hacia 
homosexuales (Yang, 1997; Davies, 2004) y (h) familiaridad con lesbianas y gays que asumen socialmente su orientación o están "fuera del clóset" (Yang, 1997). Concretamente, Adolfsen (2010) estructuró la homonegatividad en 6 dimensiones (a) Reacciones afectivas hacia la homosexualidad (b) igualdad de derechos para los homosexuales, (c) aspectos sexuales / visibilidad de la homosexualidad (d) homonegatividad moderna, reacciones afectivas hacia un amigo 0 conocido homosexual de ambos sexos y (f) aceptación de la homosexualidad en un sentido general. Si bien la investigación pasó de una visión de la homosexualidad como enfermedad a los prejuicios generados por las personas heterosexuales hacia la diversidad LGBTI [Lesbianas, Gays, Bisexuales, Transexuales e Intersexuales] (Adam, 1998; Herek, 2004; Plasek \& Allard, 1984; Plummer, 1999), esto no evitó que la definición y medida del fenómeno siga enfrentándose con dificultades. La primera es el escaso consenso teórico y terminológico sobre este tipo de actitudes. Como señala Herek (2002), han existido principalmente dos enfoques para medirlas: 1) encuestas masivas con población general relacionadas a opiniones de libertades civiles y derechos de las personas gays y 2 ) estudios de respuestas afectivas hacia la conducta de gays y lesbianas utilizando muestras de estudiantes, lo que no ha permitido una integración certera de la información. Por otra parte, el surgimiento de la denominada homonegatividad moderna -que alude a que las personas han dejado de basar sus actitudes homonegativas en valores religiosos, morales 0 tradicionales, generando una noción general más abstracta sobre la homosexualidad basada en ideas de igualdad e imparcialidad- ha generado una prevalencia de la heteronorma y los roles de género socialmente esperados en las personas (Baral et al., 2007; Cáceres et al., 2008; Harding, 2010; Adrián, 2013). Así, es posible que los heterosexuales sientan que gays y lesbianas buscan separarse de la cultura principal dominante al exagerar la importancia de su orientación sexual, deslegitimando sus demandas al considerarlas innecesarias. Esto puede conllevar la invisibilización de las discriminaciones y los crímenes de odio, que son categorizados como hechos aislados del pasado (Morrison \& Morrison, 2002; Satcher y Legget, 2006, 2007). Sin embargo, en la realidad siguen habiendo reportes acerca de los efectos adversos de la homonegatividad sobre el bienestar de gays y lesbianas (e.g. distrés, altas tasas de intentos suicidas y suicidios consumados entre adolescentes y adultos no heterosexuales) (Haas et al, 2011; Marshal et al., 2011).

Como arriba se mencionó, la medida de la homonegatividad no ha estado exenta de dificultades. Grey et al. (2013) analizaron las propiedades de 17 instrumentos cuyo propósito es medir homofobia, homonegatividad o conceptos afines (i.e. heterosexismo, homosexismo, homopositivismo, prejuicio sexual, etc.). Todas estas escalas (vid. tabla 1) utilizan el formato de autorreporte y contienen soluciones factoriales que van desde 1 a 6 factores, dependiendo de la escala. De estas, la más usada corresponde a la Subescala de Hombres Gay, perteneciente a la Escala de Actitudes hacia Lesbianas y Hombres Gays (ATLGR-R; Herek, 1988), con 66 estudios de validación, incluyendo evidencia para su forma breve.

En general, varias de las escalas que miden actitudes hacia la homosexualidad poseen mínimos criterios aceptables en su elaboración (desarrollo, muestreo, fiabilidad y validez), ciertas debilidades metodológicas (e.g. utilización erronea de ciertos criterios de selección de factores, o asumir que el alfa de Cronbach es un índice de unidimensionalidad), además de resultados disímiles en cuanto a las soluciones factoriales de cada una (si es que han sido reportadas). Por otra parte, las actitudes hacia la homosexualidad son complejas y 
multifacéticas, varían ante las pluralidades culturales y relaciones intergrupales de diferentes contextos y países en su concepción, expresión y forma (Davies, 2004; Herek, 2004; Bozon, Gayet \& Barrientos, 2009), por cuanto las validaciones de algunas escalas con muestra anglosajona (e.g. Estados Unidos y Canadá) no pueden ser implementadas en otros países sin nuevos estudios para establecer sus propiedades psicométricas específicas (Churchill, 1967; Guillemin, Bombardier \& Beaton, 1993; Bozon et al., 2009; Moreno, Herazo, Oviedo \& CampoArias, 2015). Todo lo anterior, realza la importancia de la creación y utilización de instrumentos con mayor sensibilidad para medir el constructo, así como la perentoria necesidad de su validación trans-cultural (Wu, Li \& Zumbo, 2007).

TABLA 1.

Descripción de los 17 instrumentos que miden homonegatividad,

basada en la revisión de Grey et al. (2013).

\begin{tabular}{|c|c|c|c|c|c|c|}
\hline Instrumento & Autores & Ítems & Dimensionalidad & $\begin{array}{c}\text { Confiabilidad / } \\
\text { Cronbach }\end{array}$ & $\mathrm{N}$ & Tipo de muestra \\
\hline 1. Index of Homophobia (IHP) & $\begin{array}{l}\text { (Hudson \& Ricketts, } \\
\text { 1980) }\end{array}$ & 25 & 1 factor, $r 2=60 \%$ & Total .90 & 303 & $\begin{array}{l}\text { Estudiantes } \\
\text { universitarios }\end{array}$ \\
\hline $\begin{array}{l}\text { 2. Heterosexual Attitudes } \\
\text { Toward Homosexuals (HATH) }\end{array}$ & $\begin{array}{l}\text { (Larsen, Reed, \& } \\
\text { Hoffman, 1980) }\end{array}$ & 20 & - & Total .95 & 80 & $\begin{array}{l}\text { Estudiantes } \\
\text { universitarios }\end{array}$ \\
\hline $\begin{array}{l}\text { 3. Short-Form Homosexism } \\
\text { Scale }\end{array}$ & (Hansen, 1982) & 15 & - & Total .96 & 143 & $\begin{array}{l}\text { Estudiantes } \\
\text { universitarios }\end{array}$ \\
\hline $\begin{array}{l}\text { 4. Kite Homosexuality Attitude } \\
\text { Scale }\end{array}$ & (Kite \& Deaux, 1986) & 22 & 1 factor, $r 2=42.4 \%$ & Total .93 & 569 & $\begin{array}{l}\text { Estudiantes } \\
\text { universitarios }\end{array}$ \\
\hline 5. Homophobia Scale & (Bouton et al., 1987) & 11 & 1 factor, $r 2=60.6 \%$ & Total .90 & 528 & $\begin{array}{l}\text { Estudiantes } \\
\text { universitarios }\end{array}$ \\
\hline $\begin{array}{l}\text { 6. Attitudes Toward Lesbians } \\
\text { and Gay Men-Revised (ATLG- } \\
\text { R) }\end{array}$ & (Herek, 1988) & 20 & - & Total $.89-.90$ & 368 & $\begin{array}{l}\text { Estudiantes } \\
\text { universitarios }\end{array}$ \\
\hline $\begin{array}{l}\text { 7. Attitudes Toward } \\
\text { Homosexuals }\end{array}$ & $\begin{array}{l}\text { (Altemeyer \& } \\
\text { Hunsberger, 1992) }\end{array}$ & 12 & - & Total .89 & 491 & $\begin{array}{l}\text { Padres de } \\
\text { estudiantes } \\
\text { universitarios }\end{array}$ \\
\hline 8. Homophobia Scale & $\begin{array}{l}\text { (Roese, Olson } \\
\text { Borenstein, Martin, \& } \\
\text { Shores, 1992) }\end{array}$ & 7 & - & Total .94 & 34 & $\begin{array}{l}\text { Estudiantes } \\
\text { universitarios }\end{array}$ \\
\hline $\begin{array}{l}\text { 9. Multidimensional Attitudes } \\
\text { Toward Homosexuality Scale }\end{array}$ & (LaMar \& Kite, 1998) & 49 & - & $.96-.75$ & 270 & $\begin{array}{l}\text { Estudiantes } \\
\text { universitarios }\end{array}$ \\
\hline $\begin{array}{l}\text { 10. Modern Homophobia Scale } \\
\text { (MHS) }\end{array}$ & (Raja \& Stokes, 1998) & 22 & $\begin{array}{l}3 \text { factors, } 47.3 \% \text { of } \\
\text { variance (total) }\end{array}$ & $.91-.85$ & 322 & $\begin{array}{l}\text { Estudiantes } \\
\text { universitarios }\end{array}$ \\
\hline 11. Homonegativity Scale (HS) & $\begin{array}{l}\text { (Morrison, Parriag, \& } \\
\text { Morrison, 1999) }\end{array}$ & 6 & $\begin{array}{l}1 \text { factor, } \mathrm{r} 2=53 \% \\
\text { (men)- } 55 \% \text { (women) }\end{array}$ & .84 y .88 & 1045 & $\begin{array}{l}\text { Estudiantes } \\
\text { secundaria }\end{array}$ \\
\hline 12. Homophobia Scale & $\begin{array}{l}\text { (Wright, Adams, \& } \\
\text { Bernat, 1999) }\end{array}$ & 25 & 3 factor, r $2=67.88 \%$ & Total .94 & 321 & $\begin{array}{l}\text { Estudiantes } \\
\text { universitarios }\end{array}$ \\
\hline $\begin{array}{l}\text { 13. Modern Homonegativity } \\
\text { Scale (MHS) }\end{array}$ & $\begin{array}{l}\text { (Morrison \& Morrison, } \\
\text { 2002) }\end{array}$ & 12 & $\begin{array}{l}1 \text { factor, } 45 \% \text { r2 (gay } \\
\text { version) }\end{array}$ & Total .91 & 353 & $\begin{array}{l}\text { Estudiantes } \\
\text { secundarios }\end{array}$ \\
\hline $\begin{array}{l}\text { 14. Lesbian, Gay, and Bisexual } \\
\text { Knowledge and Attitudes Scale } \\
\text { for Heterosexuals (LGB-KASH) }\end{array}$ & $\begin{array}{l}\text { (Worthington, Dillon, \& } \\
\text { Becker-Schutte, 2005) }\end{array}$ & 28 & $\begin{array}{l}5 \text { factores, } \\
\mathrm{r} 2=48,78 \%\end{array}$ & $.87-.76$ & 422 & $\begin{array}{l}\text { Listas de correos } \\
\text { electrónicos de } \\
\text { universidad e } \\
\text { Internet en general }\end{array}$ \\
\hline $\begin{array}{l}\text { 15. Homonegativity Scale- } \\
\text { Short Form }\end{array}$ & (Wrench, 2005) & 10 & 1 factor, $r 2=53.5 \%$ & .90 & 275 & $\begin{array}{l}\text { Estudiantes } \\
\text { universitarios }\end{array}$ \\
\hline $\begin{array}{l}\text { 16. Multidimensional } \\
\text { Heterosexism Inventory }\end{array}$ & (Walls, 2008) & 23 & 4 factores, $r 2=48.7 \%$ & Total .80 & 651 & $\begin{array}{l}\text { Estudiantes } \\
\text { universitarios }\end{array}$ \\
\hline $\begin{array}{l}\text { 17. Multidimensional Measure } \\
\text { of Sexual Prejudice }\end{array}$ & (Massey, 2009) & 61 & 6 factores & $.95-.83$ & 269 & $\begin{array}{l}\text { Estudiantes } \\
\text { universitarios }\end{array}$ \\
\hline
\end{tabular}


En Chile, la mayoría de los estudios sobre homosexualidad son básicamente descripciones sociodemográficas de organismos LGBTI locales y latinoamericanos (e.g. Fundación Ideas, 1997, 2001, 2003; Movimiento de Integración y Liberación Homosexual [MOVILH], 2007, 2008, 2010, 2012, 2013; Instituto Nacional de la Juventud, 2010; VIVOPOSITIVO \& ASOSIDA, 2012; Barrientos et al., 2011), donde los estudios orientados al análisis en profundidad de las propiedades psicométricas de las medidas utilizadas ha sido escaso. Nierman, Thompson, Bryan \& Mahaffey (2007), usando la escala ATLG de Herek (1994), encontraron que los chilenos presentan más prejuicios sexuales en comparación a los estadounidenses, donde algunas personas reaccionan negativamente a aquellos que violan las normas de género consideradas tradicionales, para mantener una rígida distinción entre masculinidad-feminidad (Herek, 1993b; Whitley, 2001). Estas diferencias de género son reportadas también por Barra (2002), quién mediante la creación de una escala de 18 ítems, encontró que los hombres se presentan como más homonegativos hacia la homosexualidad de ambos sexos, diferencia que, sin embargo, no está basada en la tipificación del rol sexual o predominio de rasgos personales masculinos, femeninos 0 andróginos (Bem, 1981). Además, incluso entre la población que sostiene explícitamente actitudes no homonegativas, hay una importante proporción de personas que pueden expresar de un modo implícito homonegatividad, como advierten Cárdenas \& Barrientos (2008). Estos mismos autores, trabajaron sobre la estructura de la escala ATGL (Herek, 1988), encontrando una solución de 2 factores, y un índice de fiabilidad de Cronbach de .93 (Cárdenas \& Barrientos, 2012). Hasta donde sabemos, no hay otros estudios de validaciones de escalas de homonegatividad en Chile.

Uno de los requerimientos básicos en ciencia es la medición exacta (McGrath,
2005). A pesar de ello, en la investigación psicosocial en general, los indicadores se han basado mayormente en la deducción lógica, la afirmación y la especulación, en lugar de la investigación empírica (Tucker, 2010). La continua carencia de correspondencia entre las medidas psicosociales y los constructos es un obstáculo importante para el avance del conocimiento científico, donde la exactitud representacional de los mismos resulta primordial (MacGrath, 2005). A pesar de lo anterior, la integración teórica de los resultados empíricos es aún limitada (Monroe \& Read, 2008). Los investigadores en general toman escalas ya existentes y realizan verificaciones estadísticas de validez y confiabilidad, en vez de operacionalizar constructos a través de definiciones conceptuales, desarrollar categorías y dominios, crear ítems, escalas y luego contrastar sus resultados para modificar y mejorar dichas escalas (Tucker, 2010). Además, como señala Meade \& Wright (2012) y Romero, Morera \& Wiebe (2015), diversas investigaciones que se centran en encontrar diferencias en variables demográficas a nivel observado, no contemplan la posibilidad de funcionamiento diferencial de la medida entre los grupos evaluados. La no inclusión de análisis de invarianza resultaría en una inexactitud metodológica que afecta los resultados, ya que el nivel de los datos observados no necesariamente se traduce en diferencias equivalentes en la variable latente, o el nivel de "verdad" (Thissen, Steinberg \& Gerrard, 1986), por lo que las comparaciones entre grupos deberían hacerse a nivel de variables latentes (Schmitt \& Kuljanin, 2008).

La presente investigación da cuenta de la utilización de una medida breve que resulta bastante adecuada cuando se trata de investigaciones de screening, como es el caso (Robins, Hendin \& Trzesniewski, 2001). Este formato facilita instancias de investigación que no serían posibles usando medidas extensas, al contrarrestar el 
cansancio, aburrimiento y frustración que se produce en los respondientes cuando se implementan medidas extremadamente largas y complejas (Robins et al., 2001; Saucier, 1994). Así, si los costos psicométricos de usar medidas cortas son bajos, su eficiencia relativa las vuelve herramientas atractivas de usar (Gosling, Rentfrow \& Swannet, 2003). Según 10 expuesto en referencia a las ambigüedades estructurales de las escalas de homonegatividad, el propósito de este estudio es realizar un análisis psicométrico en profundidad y aportar evidencias a favor o en contra de la validez de la Escala Breve de Homonegatividad (Wrench, 2005) en el marco de su aplicación en Chile. Si bien existen multitud de estudios "de validación" de escalas de homonegatividad, la mayor parte de ellos se han limitado a una aproximación puramente exploratoria (y en consecuencia, fundamentalmente mecánica, con las limitaciones metodológicas $y$ teóricas que ello implica). También, sorprendentemente, en la mayor parte de los casos se tiende a obviar aspectos fundamentales de la validez, tales como la invarianza de medida entre grupos cuya comparación es interesante desde un punto de vista teórico. El objetivo de esta investigación es contribuir al estudio de la validez de la medida de la homonegatividad, mediante un análisis en profundidad de las propiedades de uno de los instrumentos para su medida.

\section{MÉTODO}

\section{Participantes}

Fue utilizada una muestra de 303 personas (71\% mujeres, $29 \%$ hombres) mediante un muestreo por conveniencia tipo "bola de nieve" (Ver Tabla N²). La toma de datos se realizó en 2014 mediante la plataforma SurveyMonkeyTM, sitio web destinado a realizar encuestas on-line por internet. Se decidió recoger los datos vía cuestionario web ya que esto permite alcanzar una gran muestra de forma rápida. Por otra parte, existe evidencia fuerte de que los datos basados en internet no difieren a los de una aplicación presencial, que la población con acceso a internet que responde cuestionarios online es diversa demográficamente, se encuentra motivada a responder y está dentro de los parámetros normales de ajuste psicológico (Gosling et al., 2004).

\section{TABLA 2.}

Caracterización de la muestra.

\begin{tabular}{|c|c|c|}
\hline Variable & Categoría & $\begin{array}{c}\text { Porcentaje, } \\
\text { Rango o } \\
\text { Media }\end{array}$ \\
\hline \multirow{3}{*}{ Sexo } & Hombre & $89(29,4 \%)$ \\
\hline & Mujer & $214(70,6 \%)$ \\
\hline & Media & 23,2 años \\
\hline \multirow[t]{2}{*}{ Edad } & Rango & $18-53$ \\
\hline & DS & 5,5 \\
\hline \multirow{4}{*}{$\begin{array}{l}\text { Pertenece a } \\
\text { una etnia }\end{array}$} & $\mathrm{Si}$ & $3 \%$ \\
\hline & No & $97 \%$ \\
\hline & Sin instrucción & $0 \%$ \\
\hline & Educación básica & $0 \%$ \\
\hline Nivel de & Educación media & $43(14,2 \%)$ \\
\hline \multirow[t]{3}{*}{ estudios } & Educación media superior & $44(14,2 \%)$ \\
\hline & Educación superior & $206(68 \%)$ \\
\hline & Postgrado (maestría/doctorado) & $11(3,6 \%)$ \\
\hline Haber & $\mathrm{Si}$ & $35 \%$ \\
\hline $\begin{array}{l}\text { recibido } \\
\text { clases de } \\
\text { sexualidad } \\
\text { humana }\end{array}$ & $\begin{array}{l}\text { sexualidad } \\
\text { humana }\end{array}$ & $65 \%$ \\
\hline Conocer & $\mathrm{Si}$ & $97,70 \%$ \\
\hline \multicolumn{3}{|l|}{ e a una } \\
\hline \multicolumn{3}{|l|}{$\begin{array}{l}\text { persona gay } \\
\text { o lesbiana }\end{array}$} \\
\hline \multirow{5}{*}{$\begin{array}{l}\text { Orientación } \\
\text { sexual }\end{array}$} & Heterosexual & $73,60 \%$ \\
\hline & Homosexual & $15,20 \%$ \\
\hline & Bisexual & $8,90 \%$ \\
\hline & Asexual & $0,70 \%$ \\
\hline & Otra o n/r & $1,70 \%$ \\
\hline
\end{tabular}

\section{Instrumentos}

Encuesta de información demográfica: Se consultó acerca de la edad, sexo, nivel educativo, el haber cursado materias relacionadas con sexualidad humana, diversidad sexual o similares, pertenencia a alguna etnia indígena, el conocer personalmente a alguna persona homosexual (gay o lesbiana) y la orientación sexual del respondiente, todas variables que según la literatura, inciden en la expresión de la homonegatividad. 
Escala Breve de Homonegatividad: Esta medida creada por Wrench (2005) posee 10 ítems (Ver Tabla $\mathrm{N}^{\circ} 3$ ) con cinco categorías de respuesta de 1 a 5 , desde Muy en Desacuerdo a Muy de Acuerdo, que mide las percepciones individuales negativas hacia sujetos gays, lesbianas y bisexuales. Estos reactivos fueron extraídos de una anterior versión de la escala de 16 ítems (Wrench, 2001). El autor tomó como base teórica la comparación entre las escalas tradicionales (old-fashioned) y las escalas de homonegatividad moderna propuesta por Morrison \& Morrison (2002), La muestra original estuvo compuesta por 275 estudiantes universitarios en cursos de comunicación en St. Clairsville, Ohio (165 hombres, 108 mujeres y 2 sujetos que no reportaron sexo). Wrench (2005) realizó un AFC que arrojó una solución unifactorial que explicaría el $53.5 \%$ de la varianza, además de una fiabilidad de .90 mediante alfa de Cronbach. Conjuntamente, se realizaron pruebas de validez concurrente, encontrando un $r=.40$ entre homonegatividad y fundamentalismo religioso y un $r=.56$ entre homonegatividad y etnocentrismo.

TABLA 3.

Escala Breve de Homonegatividad (Wrench, 2005) en su versión original y en español.

\begin{tabular}{ll}
\hline \multicolumn{1}{c}{ Versión en inglés } & \multicolumn{1}{c}{ Versión en español } \\
\hline 1.Gays and lesbian people make me nervous & 1. Las personas gays y lesbianas me ponen nervioso(a) \\
2. Homosexuality is perfectly normal & 2. La homosexualidad es completamente normal \\
3. I wouldn't want to have gay or lesbian friends & 3. No podría tener amigos gays o amigas lesbianas \\
4. I would trust a gay or lesbian person & 4. Podría confiar en una persona gay o lesbiana \\
5. I fear homosexual persons will make sexual advances towards me & 5. Me atemoriza que homosexuales me hagan insinuaciones sexuales \\
6. I would have no problem living with someone who is gay or lesbian & 6. No tendría problema viviendo con una persona gay o lesbiana \\
7. Homosexual behavior should be perfectly legal & 7. El comportamiento homosexual debería ser visto como algo normal. \\
8. I would have a serious problem if I saw two men or women kissing in public & 8. Me molestaría ver a dos hombres o dos mujeres besarse en público, \\
9. I think that gay and lesbian people need civil rights protection & 9. Los gays y lesbianas necesitan protección de sus derechos civiles \\
10. When I see a gay or lesbian person I think, "what a waste". & 10. Cuando veo a un gay o una lesbiana pienso, "qué desperdicio" \\
\hline
\end{tabular}

Cuestionario PANAS Short Form: Se trata de un cuestionario para evaluar la presencia de emociones positivas y negativas en el respondiente. El original fue desarrollado por Watson, Clark \& Tellegen (1988). Constaba de 60 ítems y medía Afecto 0 Emocionalidad Positiva y Negativa. La versión utilizada en este estudio corresponde a la traducción del trabajo de Thompson (2007), quién creó una versión abreviada de 10 ítems. En su validación en inglés con una muestra de 1789 sujetos, se obtuvo que la confiabilidad interna, la invariancia factorial intercultural y validez convergente y de criterio son psicométricamente aceptables (Thompson, 2007). En Chile ha sido validada la versión de 20 ítems, obteniéndose indicadores óptimos de confiabilidad, consistencia interna y validez factorial y externa (Dufey \& Fernández, 2012; Robles \& Páez, 2003). Todos los ítems son tipo Likert de 5 opciones y puntaje directo (desde Muy en desacuerdo a Muy de acuerdo).

\section{Requerimientos éticos}

La presente investigación está adscrita al código de conducta del Psicólogo de la APA (2010) referentes a realizar investigación. Por las características anónimas de esta investigación, podría haberse prescindido de un consentimiento informado propiamente tal (artículo 8.07 del código citado). Pero aun así, en la presentación de la página web de aplicación de los 
instrumentos, se redactó un consentimiento que contenía la presentación de los investigadores, el propósito de la investigación, la duración estimada, los procedimientos, la total confidencialidad de los datos obtenidos e información de contacto en caso de requerir información 0 dudas al respecto, mediante correo electrónico. Además, al finalizar este estudio, los investigadores realizaron un compromiso de realizar una devolución de la información a modo general a través de correo electrónico, mediante un breve informe ejecutivo de los resultados encontrados.

\section{Procedimiento}

Se utilizaron la Escala Breve de Homonegatividad (Wrench, 2005) y el cuestionario PANAS Short Form. Para su traducción al español se utilizó el método de retro-traducción (Muñiz, Elosua \& Hambleton, 2013) por parte de tres traductores con conocimiento elevado del idioma inglés. Posteriormente, se estableció una cuenta en SurveyMonkey ${ }^{T M}$, página web diseñada para el desarrollo de encuestas online, donde las escalas fueron digitalizadas y configuradas para su óptima utilización (insertar los ítems, diseñar la página, configurar recopiladores de respuesta). Se distribuyeron los links a través de redes sociales (Facebook, Twitter, foros de discusión chilenos) y correos electrónicos. A su vez, se pidió a los respondientes compartir los enlaces webs entre sus propios contactos, para poder captar la mayor cantidad de sujetos. Finalmente, al cabo de dos semanas, se cerró el recopilador y se conformaron las bases de datos para su análisis.

\section{Plan de Análisis de los datos}

En primer lugar, se depuró la base de datos, eliminando sujetos que respondieron sólo la encuesta de información demográfica, dejando sin responder los demás instrumentos, resultando así una muestra de 303 casos válidos para analizar. Después de la primera depuración, no se encontró ningún caso con datos perdidos.

En segundo lugar, se establecieron pruebas estadísticas descriptivas con el fin de caracterizar demográficamente la muestra obtenida.

En tercer lugar, se efectuó un análisis factorial exploratorio (AFE), mediante el programa FACTOR 9.2 (Lorenzo-Seva y Ferrando, 2006) con el propósito de elaborar una primera aproximación a la estructura dimensional de la Escala Breve de Homonegatividad (Wrench, 2005).

En cuarto lugar se realizó un Análisis factorial confirmatorio (AFC), mediante el programa Mplus 7.3, (Muthen \& Muthen, 2014), con el propósito de determinar el número de factores óptimos que posee la escala de Homonegatividad que expliquen de mejor forma la varianza compartida por los ítems.

En quinto lugar, se llevó a cabo un análisis de invarianza de medida entre hombres y mujeres, mediante la clasificación clásica de Meredith (1993); en este paso se comprobó la invarianza de configuración, de las cargas factoriales (invarianza débil), y de los umbrales (invarianza fuerte).

En sexto lugar, se estimó el impacto de las emociones en el momento de responder la escala sobre las puntuaciones de homonegatividad, mediante la estimación del un modelo de ecuaciones estructurales.

\section{RESULTADOS}

\section{Análisis Factorial Exploratorio}

Las pruebas de normalidad univariada indicron alta asimetría y curtosis, por lo que se viola el principio de normalidad. La prueba de Mardia indica violación del principio de normalidad multivariada en asimetría (coeficiente $=41.38$; estadístico= 2238.38; $\quad g l=220 ; \quad p=1) \quad$ y curtosis 
(coeficiente $=191.36 ; \quad$ estadístico $=40.02 ;$ $p<.05)$. Analizando la matriz de correlaciones entre items, se evidencia que todos los valores son >.3, por lo que se recomienda seguir adelante el análisis. Se revisó la pertinencia de la solución factorial, es decir, la existencia de una estructura de relaciones entre los elementos idónea para realizar la extracción de factores. En ese sentido, la prueba Kaiser- Meyer- Olkin alcanza un valor adecuado (KMO $=.89)$, lo que indica que el set de datos es adecuado para su tratamiento mediante análisis factorial exploratorio. El test de esfericidad de Barlett también arrojó valores adecuados $\left(X^{2}=1030.9 ; g l=45 ; p<.01\right) . \quad Y$ el determinante de la matriz alcanzó un valor de .03, donde lo esperado es un valor entre 0 y 1 pero pequeño (Gorsuch, 1983; Pett et al., 2003; citados por Méndez \& Rondón, 2012). Todo lo anterior indicó que la estructura es la adecuada y sí se justifica la realización del AFE.

Así, se llevó a cabo el AFE sobre los 10 ítems de la Escala de Homonegatividad en la muestra chilena. Se efectuaron los cálculos mediante la matriz de correlaciones policóricas, recomendada por Muthén \& Kaplan (1992) cuando la distribución de los ítems son asimétricas o con exceso de curtosis. Como método de extracción de factores, se usó el método de mínimos cuadrados ponderados (ULS). Este método es el más apropiado cuando el principio de normalidad no se cumple (Ximénez \& García, 2005). El método de rotación usado fue el oblicuo de PROMAX, debido a que cambia los resultados de una rotación ortogonal hasta establecer una solución con cargas factoriales lo más cercana a la "estructura ideal". Este método es recomendado cuando se presume que los nuevos factores pudiesen estar correlacionados entre sí (Méndez \& Rondón, 2012), como sería el caso de la Escala Breve de Homonegatividad. La regla de Kaiser Guttman (retener tantos factores como valores propios superiores a 1.00 aparezcan) sugiere la retención de 1 factor. Para verificarlo, se optó por realizar un análisis paralelo optimizado (Timmerman \& Lorenzo-Seva, 2011) como criterio de decisión, ya que se ha comprobado en diversas ocasiones que corresponde método más fiable para decidir el número de factores a retener (Abad, Olea, Ponsoda \& García, 2010), arrojando una solución óptima de 1 factor para la escala, que explica el $60,1 \%$ de la varianza común (Ver Tabla N4).

En cuanto a la confiabilidad de la escala, el índice de Alfa de Cronbach alcanzó un valor de .91, considerado excelente, lo que concuerda con el análisis psicométrico del propio Wrench (2005) para la escala en inglés (alfa =.90).

TABLA 4.

Cargas factoriales del análisis factorial exploratorio (solución unidimensional).

\begin{tabular}{lcc}
\hline \multicolumn{1}{c}{ Ítems } & Carga & Comunalidad \\
\hline 1. Las personas gays y lesbianas me ponen nervioso(a) & 0.736 & 0.542 \\
2. La homosexualidad es completamente normal & 0.807 & 0.651 \\
3. No podría tener amigos gays o amigas lesbianas & 0.782 & 0.612 \\
4. Podría confiar en una persona gay o lesbiana & 0.662 & 0.438 \\
5. Me atemoriza que homosexuales me hagan insinuaciones sexuales & 0.604 & 0.365 \\
6. No tendría problema viviendo con una persona gay o lesbiana & 0.752 & 0.566 \\
7. El comportamiento homosexual debería ser visto como algo normal & 0.827 & 0.684 \\
8. Me molestaría ver a dos hombres o dos mujeres besarse en público, más que a una pareja de hombre y mujer & 0.761 & 0.579 \\
9. Los gays y lesbianas necesitan protección de sus derechos civiles & 0.719 & 0.516 \\
10. Cuando veo a un gay o una lesbiana pienso, "qué desperdicio" & 0.531 & 0.282 \\
\hline
\end{tabular}




\section{Análisis Factorial Confirmatorio}

Dados los resultados anteriores, se realizó un AFC en la Escala Breve de Homonegatividad (Wrench, 2005) sobre la solución unidimensional. Se utilizó como método de estimación WLSMV (mínimos cuadrados no ponderados con ajuste de medias y varianzas) dada la naturaleza ordinal de los datos de entrada. En las tablas $\mathrm{N}^{\circ} 5$ y N $\mathrm{N}^{\circ} 6$ se reportan los índices de ajuste del modelo unidimensional y las cargas factoriales, respectivamente. El modelo presentó índices de ajuste adecuados. En cuanto a las cargas factoriales, el factor estuvo adecuadamente representado por sus ítems (cargas mayores a .30), y los errores estándar fueron bajos. Dado el correcto ajuste del modelo unidimensional, tanto en AFE como en AFC, no se consideró necesaria la comprobación de modelos menos parsimoniosos (i.e., con más factores).

\section{TABLA 5.}

Indicadores análisis factorial confirmatorio (solución unidimensional).

\begin{tabular}{lc}
\hline Indicadores AFC & Nivel \\
\hline Chi cuadrado & 118,75 \\
gl & 35 \\
RMSEA & 0,069 \\
Cfi & 0,97 \\
Tli & 0,96 \\
\hline
\end{tabular}

TABLA 6.

Cargas factoriales extraídas mediante análisis factorial confirmatorio.

\begin{tabular}{lrc}
\hline \multicolumn{1}{c}{ Ítems } & Carga & $\mathrm{R}^{2}$ \\
\hline 1. Las personas gays y lesbianas me ponen nervioso(a) & 0.736 & 0.541 \\
2. La homosexualidad es completamente normal & 0.874 & 0.763 \\
3. No podría tener amigos gays o amigas lesbianas & 0.802 & 0.643 \\
4. Podría confiar en una persona gay o lesbiana & 0.614 & 0.377 \\
5. Me atemoriza que homosexuales me hagan insinuaciones sexuales & 0.630 & 0.397 \\
6. No tendría problema viviendo con una persona gay o lesbiana & 0.734 & 0.538 \\
7. El comportamiento homosexual debería ser visto como algo normal. & 0.877 & 0.769 \\
8. Me molestaría ver a dos hombres o dos mujeres besarse en público, más que a una pareja de hombre y mujer & 0.774 & 0.599 \\
9. Los gays y lesbianas necesitan protección de sus derechos civiles & 0.692 & 0.479 \\
10. Cuando veo a un gay o una lesbiana pienso, "qué desperdicio" & 0.516 & 0.267 \\
\hline
\end{tabular}

\section{Invarianza de medida}

En el siguiente paso se puso a prueba la invarianza del modelo unidimensional, en base al sexo de los respondientes. Demostrar la invarianza es un requisito crucial para habilitar cualquier comparación entre grupos (Marsh et al, 2010). Se siguió el procedimiento clásico (Meredith, 1993). Este procedimiento contempla la puesta a prueba de al menos tres niveles de invarianza, con restrictividad creciente: en el primer paso (invarianza configural), se pone a prueba la hipótesis de si en los grupos comparados el modelo se configura en el mismo número de factores. En la invarianza débil, se comprueba si en ambos grupos las cargas factoriales son equivalentes entre los grupos comparados; si se confirma este tipo de invarianza, se puede razonablemente deducir que en ambos grupos el constructo latente estudiado adquiere un significado similar. En la invarianza fuerte, se exige que las interceptas de los items (o umbrales en el caso que nos ocupa) sean equivalentes; la violación de esta invarianza puede suponer la existencia de funcionamiento diferencial monotónico de algunos ítems. 
Por último, la invarianza estricta implica, además del resto de invarianzas, que los residuos de los ítems sean iguales en ambos grupos; el incumplimiento de la invarianza estricta puede suponer diferencias en la fiabilidad de la escala (0 sus ítems) entre ambos grupos.

TABLA 7.

Invarianza de medida.

\begin{tabular}{cccc}
\hline Indicador & Configural & Débil & Fuerte \\
\hline Chi cuadrado & 335,45 & 445,24 & 897,42 \\
gl & 108 & 111 & 121 \\
RMSEA & 0,072 & 0,079 & 0,134 \\
CFI & 0,95 & 0,92 & 0,86 \\
TLI & 0,96 & 0,93 & 0,87 \\
\hline
\end{tabular}

Como se puede observar en la tabla $\mathrm{N}^{\circ} 7$, los resultados apoyan la existencia de invarianza configural y débil; los niveles de ajuste fueron pobres para la invarianza fuerte, con lo que no se consideró necesario comprobar la invarianza estricta. Si bien la estructura de la escala y el significado del factor homofobia son las mismas entre sexos, cabe la posibilidad de que exista funcionamiento diferencial de algunos ítems.

TABLA 8.

Ajuste del modelo estructural de homonegatividad y emocionalidad.

\begin{tabular}{cc}
\hline Indicador & Nivel \\
\hline Chi sq & 375,07 \\
df & 167 \\
rmsea & 0,045 \\
CFI & 0,98 \\
TLI & 0,98 \\
\hline
\end{tabular}

Efecto de la emocionalidad sobre las respuestas de homonegatividad

Se puede señalar del modelo que, en general, las influencias de las 2 dimensiones de Emocionalidad de la Escala PANAS en los resultados de la Homonegatividad de la muestra son débiles y no significativas, donde la Emocionalidad Positiva manifestada por el respondiente, posee un valor explicativo de $1.27 \%$ de la varianza para la Homonegatividad y Emocionalidad negativa un valor explicativo inferior al $1 \%$ (Ver Tabla $N^{\circ} 8$ y Figura $\left.N^{\circ} 1\right)$, por lo que se concluye que el estado emocional del respondiente al momento de responder no afectaría las respuestas a la Escala de Homonegatividad. Ello implica que la evaluación de la actitud no estaría afectada por estados de ánimo temporales. 
FIGURA 1.

Modelo estructural de la variable dependiente Homonegatividad y su relación con los 2 factores de la Escala Panas de Emocionalidad (Emocionalidad Positiva y Negativa).

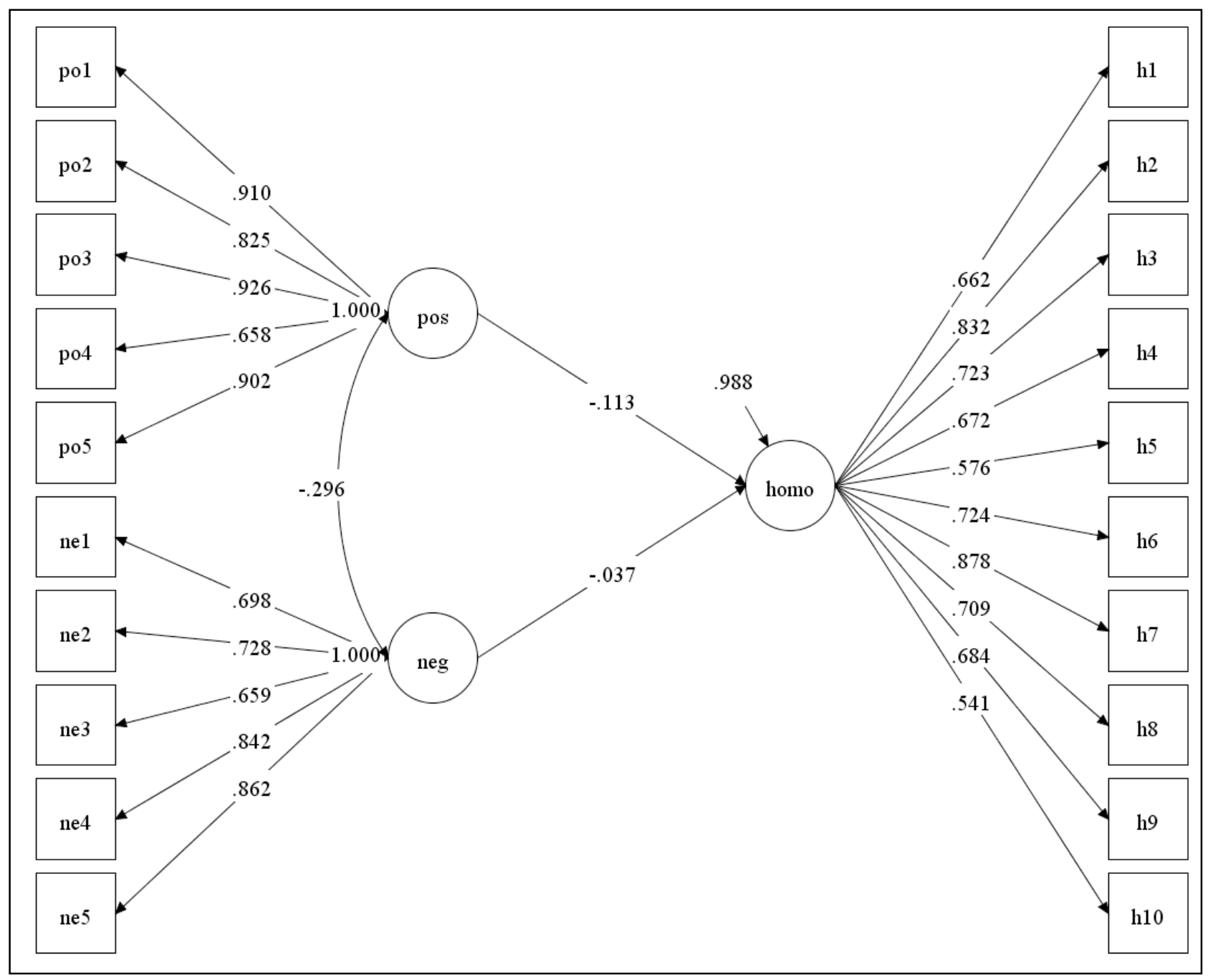

Nota: pos=emocionalidad positiva; neg=emocionalidad negativa; homo=homonegatividad

\section{DISCUSIÓN}

En la presente investigación basada en la web sobre homonegatividad, se llevó a cabo la validación de la Escala Breve de Homonegatividad (Wrench, 2005) en una muestra Chilena. Para contrastar la evidencia de los indicadores psicométricos de la Escala reportadas por el autor (Wrench, 2005) y para comprobar si el instrumento realmente evaluaba la homonegatividad -concebida como las percepciones individuales negativas hacia sujetos gays, lesbianas y bisexuales- se realizó primeramente un Análisis Factorial Exploratorio que sugirió un comportamiento unifactorial de la escala. Posteriormente, a través de un Análisis Factorial Confirmatorio, se verificó que un solo factor es suficiente para explicar la mayor parte de la variabilidad presente en las respuestas de los sujetos. Así, la propuesta de la versión original en inglés se ve apoyada al ser aplicada en una población culturalmente distinta, obteniéndose adecuados índices de ajuste. 
En cuanto a su confiabilidad, la escala presenta indicadores de Alfa de Cronbach considerados óptimos, al tomar en cuenta los datos (alfa= .91), alcanzando niveles similares de confiabilidad a los reportados por Wrench (2005) en el instrumento original (alfa $=.90)$, comprobando la adecuada fiabilidad del instrumento en diversos contextos de aplicación.

Sin embargo, la escala no alcanzó un nivel de invarianza fuerte cuando se realizó la comparación en base al género del respondiente. Ello implica que no es posible la comparación de puntuaciones directas entre hombres y mujeres, y sugiere la presencia de funcionamiento diferencial del item (i.e., algunos items podrían estar monotónicamente sesgados a favor de uno de los dos sexos). Esto es una amenaza grave a la validez de la medida, en cuanto implica que la homonegatividad está siendo medida de forma distinta entre hombres y mujeres. No implica sin embargo que toda la escala esté sesgada, sino posiblemente sólo alguno de sus items. Ello sugiere la necesidad de estudios integramente dedicados al análisis del funcionamiento diferencial del item, por ejemplo mediante métodos encuadrados en la Teoría de la Respuesta al Item. Es por tanto necesario que futuras investigaciones dedicadas a la creación de escalas de homonegatividad tengan en cuenta el posible funcionamiento diferencial como base para una adecuada selección de items.

Por otra parte, en base al modelo estructural entre Emocionalidad (reportado por la Escala PANAS) y Homonegatividad, tampoco se encontraron influencias relevantes, por lo que se confirma, que al menos para esta escala, el estado emocional del encuestado al responder no afecta sus respuestas sobre percepciones negativas dirigidas hacia la homosexualidad. Ello es sin duda una evidencia a favor de la validez de la escala, entendida como la estabilidad de la actitud a medir, que no es dependiente de estados emocionales de carácter temporal.

Tomando en cuenta las limitaciones con las que el presente estudio se encuentra está el tipo de muestra, debido al tipo de muestreo (no probabilístico) y a la toma de datos vía web, que si bien permiten ampliar el tamaño muestral, sesga los resultados en cuanto a que sólo personas con acceso a internet contestan la batería de instrumentos. Tal como señala Hernández, Fernández y Baptista (2003), con este tipo de muestreo los datos no se pueden generalizar a las poblaciones con seguridad. El que el muestreo haya sido por bola de nieve, también sesga la información, en cuanto las personas realizan una selección totalmente arbitraria sobre a quién le comparte el enlace a la encuesta, generalmente personas con las que tienen afinidad y que se asemejen a ellos mismos en alguna característica específica.

Ahora bien, a modo de sugerencias hacia futuras intervenciones con la misma escala, se puede recomendar que el estudio sea replicado mediante una metodología de toma de muestras presenciales de papel y lápiz, preferentemente de forma aleatoria, para evaluar si el formato de evaluación influye en las respuestas y por ende en el nivel de homonegatividad. También sería conveniente complementar con algún instrumento que mida Deseabilidad Social y/o Homofobia Implícita, como lo hicieron Barrientos \& Cárdenas en Chile (2008), ya que ésta puede afectar las indagaciones sobre homonegatividad en las que se cuestiona la identidad social, provocando discriminación (Marín \& Marín, 1991).

Además, tomando en cuenta que las actitudes son susceptibles a cambiar con el tiempo, realizar estudios de tipo longitudinal puede proporcionar una manera de mostrar este dinamismo en diferentes grupos de individuos a lo largo de sus vidas. Así también, conseguir muestras de mayor tamaño y potenciar el uso de medidas 
objetivas de homonegatividad (p. ej. indicadores de comportamiento) pueden aumentar la fiabilidad de los resultados (Moreno et al., 2015). En este sentido, resultaría interesante poder estudiar las historias de aprendizaje de la sexualidad de cada respondiente, sus experiencias personales e incluso las actitudes hacia la homosexualidad de padres y pares de los respondientes, para intentar medir la influencia social que tiene el medio en la expresión de la homonegatividad. En la misma línea, las investigaciones deberían examinar los cambios actitudinales en culturas que tradicionalmente han diferido en expresar la homonegatividad, con el fin de analizar posibles causas que expliquen, por ejemplo, la discrepancia entre los altos niveles de homonegatividad de estudiantes universitarios heterosexuales versus las evidencias de algunas investigaciones que sugieren que la homonegatividad sería menor en las generaciones de adolescentes actuales (Savin-Williams, 2005).

Por otra parte, sería interesante realizar una aplicación de la Escala Breve de Homonegatividad a personal de servicios, ya que han existido estudios que afirman que estudiantes ligados al área de prestaciones de servicios de salud 0 sociales, han reportado altos niveles de actitudes negativas hacia las minorías sexuales en respuestas a otros instrumentos de Homonegatividad (e.g. Campo \& Herazo, (2013); Moral, Valle \& Martínez, 2013).

Finalmente, y en un lineamiento más aplicado, el desarrollo de potenciales intervenciones para el cambio de actitud se sugiere con el fin de prevenir el acoso escolar y evitar restringir el acceso a recursos de salud debido a las actitudes negativas hacia las personas gays $y$ lesbianas en muchos contextos (Moreno et al., 2015).

\section{REFERENCIAS}

Abad, F. J., Olea, J., Ponsoda, V. y García, C. (2011). Medición en ciencias sociales y de la salud. Madrid: Síntesis.

Adam, B. D. (1998). Theorizing homophobia. Sexualities, 1(4), 387404.

Adolfsen, A., ledema, J., \& Keuzenkamp, S. (2010). Multiple dimensions of attitudes about homosexuality: development of a multifaceted scale measuring attitudes toward homosexuality. Journal of Homosexuality, 57(10), 1237-1257. http://doi.org/10.1080/00918369.2010.5 17069

Adrián, T. (2013). Cuadrando el círculo: despatologización vs derecho a la salud de personas trans en DSM-5 y CIE-11. Comunidad y Salud, 11.

Baral, S., Sifakis, F., Cleghorn, F. \& Beyrer, C. (2007). Elevated risk for HIV infection among men who have sex with men in low- and middle-income countries 2000-2006: A systematic review. PLOS Medicine, 4, 1901-1911. doi:10.1371/journal.pmed.0040339

Barra, E. (2002). Influencia del sexo v de la tipificación del rol sexual sobre las actitudes hacia la homosexualidad masculina y femenina. Revista latinoamericana de Psicología, 34(3), 275-284.

Barrientos, J. E., \& Cárdenas, M. (2012). A confirmatory factor analysis of the Spanish language version of the Attitudes Toward Lesbians and Gay Men Scale (ATLG). Universitas Psychologica, 11(2), 579-586.

Barrientos, J., Díaz, J., \& Muñoz, F. (2011). Derechos, política y diversidad sexual. Segunda encuesta marcha por la diversidad sexual, Santiago, Chile.

Bayer, R. (1981). Homosexuality and American psychiatry: The politics of diagnosis. Princeton University Press.

Bell, N. (1989). AIDS and women: Remaining ethical issues. AIDS Education and Prevention, 1, 22-30 
Bem, S. L. (1981). Gender schema theory: A cognitive account of sex typing. Psychological review, 88(4), 354.

Bhugra, D. (1987). Homophobia: A review of the literature. Sexual and marital therapy, 2(2), 169-177.

Borrillo, D. (2001). Homofobia. Barcelona: Bellaterra.

Bozon, M., Gayet, C., \& Barrientos, J. (2009). A life course approach to patterns and trends in modern Latin American sexual behavior. JAIDS Journal of Acquired Immune Deficiency Syndromes, 51, S4-S12.

Cáceres, C. F., Pecheny, M., Frasca, T., Rios, R. R., \& Pocahy, F. (2008). Review of legal frameworks and the situation of human rights related to sexual diversity in low and middle income countries. Sexually Transmitted Infections, 84, i49-i56.

Campo, A. \& Herazo, E. (2013). Homofobia en estudiantes de trabajo social. Salud Uninorte, 29(1), 96-103.

Cárdenas, M., \& Barrientos, J. E. (2008). The Attitudes toward Lesbians and Gay Men Scale (ATLG): Adaptation and testing the reliability and validity in Chile. Journal of Sex Research, 45(2), 140-149.

Carneiro, N. S. (2009). Homossexualidades: Uma psicologia entre ser, pertencer e participar. Livpsic.

Chamberland, L., \& Lebreton, C. (2012). Réflexions autour de la notion d'homophobie: succès politique, malaises conceptuels et application empirique. Nouvelles questions féministes, 31(1), 27-43.

Churchill, W. (1967). Homosexual behavior among males: A cross-cultural and cross species investigation. Hawthorn Books.

Clarke, V., Ellis, S. J., Peel, E., \& Riggs, D. W. (2010). Lesbian, gay, bisexual, trans and queer psychology: An introduction. Cambridge University Press.

Costa, A. B., Bandeira, D. R., \& Nardi, H. C. (2013). Systematic review of instruments measuring homophobia and related constructs. Journal of Applied Social Psychology, 43(6), 1324-1332.

http://doi.org/10.1111/jasp.12140

Currie, M. R., Cunningham, E. G., \& Findlay, B. M. (2004). The Short Internalized Homonegativity Scale: Examination of the factorial structure of a new measure of internalized homophobia. Educational and Psychological Measurement, 64(6), 1053-1067.

Davies, M. (2004). Correlates of negative attitudes toward gay men: Sexism, male role norms, and male sexuality. Journal of Sex Research, 41, 259-266.

Dermer, S. B., Smith, S. D., \& Barto, K. K. (2010). Identifying and correctly labeling sexual prejudice, discrimination, and oppression. Journal of counseling \& development, 88(3), 325-331.

Dufey, M., \& Fernández, A. M. (2012). Validez y confiabilidad del Positive Affect and Negative Affect Schedule (PANAS) en estudiantes universitarios chilenos. Revista Iberoamericana de Diagnóstico y Evaluación Psicológica, 34(2), 157-173.

Fundación Ideas (1997). Primera Encuesta de Tolerancia y No Discriminación. Santiago, Chile.

Fundación Ideas (2001). Segunda Encuesta de Tolerancia y No Discriminación. Santiago, Chile.

Fundación Ideas (2003). Tercera Encuesta de Tolerancia y No Discriminación. Santiago, Chile.

Fyfe, B. (1983). "Homophobia" or homosexual bias reconsidered. Archives of Sexual Behavior, 12(6), 549-554.

Gato, J., Fontaine, A. M., \& Carneiro, N. S. (2012). Multidimensional scale of attitudes toward lesbians and gay Men: Construction and preliminary validation. Paidéia, 22(51), 11-20. http://doi.org/10.1002/casp.669

Gosling, S. D., Rentfrow, P. J., \& Swann, W. B. (2003). A very brief measure of the Big-Five personality domains. Journal 
of Research in personality, 37(6), 504528.

Gosling, S. D., Vazire, S., Srivastava, S., \& John, O. P. (2004). Should we trust web-based studies? A comparative analysis of six preconceptions about internet questionnaires. American Psychologist, 59(2), 93.

Gray, C., Russell, P., \& Blockley, S. (1991). The effects upon helping behaviour of wearing pro-gay identification. British Journal of Social Psychology, 30, 171178.

Grey, J. A., Robinson, B. B. E., Coleman, E., \& Bockting, W. O. (2013). A systematic review of instruments that measure attitudes toward homosexual men. Journal of Sex Research, 50(3-4), 32952.

http://doi.org/10.1080/00224499.2012.7 46279

Guillemin, F., Bombardier, C., \& Beaton, D. (1993). Cross-cultural adaptation of health-related quality of life measures: Literature review and proposed guidelines. Journal of Clinical Epidemiology, 46, 1417-1432. Doi:10.1016=0895-4356(93)90142-N

Haaga, D. A. (1991). "Homophobia"?. Journal of Social Behavior \& Personality.

Haas, A. P., Eliason, M., Mays, V. M., Mathy, R. M., Cochran, S. D., D'Augelli, A. R., Silverman, M. M., Fisher, P. W., Hughes, T., Rosario, M., et al. (2011). Suicide and suicide risk in lesbian, gay, bisexual, and transgender populations: Review and recommendations. Journal of Homosexuality, 58(1), 10-51.

Harding, R. (2010). Regulating sexuality: Legal consciousness in lesbian and gay lives. Routledge.

Herek, G. M. (1984). Attitudes toward lesbians and gay men: A factor-analytic study. Journal of homosexuality, 10(12), 39-51.

Herek, G. M. (1988). Heterosexuals' attitudes toward lesbians and gay men: Correlates and gender differences.
Journal of Sex Research, 25(4), 451477.

Herek, G. M. (1993). The context of antigay violence: Notes on cultural and psychological heterosexism. En L. Garnets \& D. Kimmel (Eds.), Psychological perspectives on lesbian and gay male experiences (pp. 90107). New York: Columbia University Press.

Herek, G. M. (2002). Gender gaps in public opinion about lesbians and gay men. Public Opinion Quarterly, 66(1), 40-66.

Herek, G. M. (2004). Beyond "homophobia": Thinking about sexual prejudice and stigma in the twenty-first century. Sexuality Research and Social Policy, 1(2), 6-24.

Herek, G. M., \& Gonzalez-Rivera, M. (2006). Attitudes toward homosexuality among US residents of Mexican descent. Journal of sex research, 43(2), 122-135.

Hudson, W. W., \& Ricketts, W. A. (1980). A strategy for the measurement of homophobia. Journal of homosexuality, 5(4), 357-372.

Instituto Nacional de la Juventud (2010). Sexta Encuesta Nacional de Juventud. Santiago, Chile

Kite, M. E., \& Whitley, B. E., Jr. (1996). Sex differences in attitudes toward homosex- ual persons, behaviors, and civil rights. A meta-analysis. Personality and Social Psychology Bulletin 22(4), 336-353.

Lewis, G. B. (2003). Black-white differences in attitudes toward homosexuality and gay rights. Public Opinion Quarterly, 67, 59-78.

Logan, C. R. (1996). Homophobia? No, homoprejudice. Journal of homosexuality, 31(3), 31-53.

Lottes, I. L., \& Grollman, E. A. (2010). Conceptualization and assessment of homonegativity. International Journal of Sexual Health, 22(4), 219-233.

MacDonald, A. P., Huggins, J., Young, S., \& Swanson, R. A. (1973). Attitudes toward homosexuality: Preservation of 
sex morality or the double standard?. Journal of Consulting and Clinical Psychology, 40(1), 161.

Marin, G., \& Marin, B. V. (1991). Research with Hispanic populations. Sage Publications, Inc.

Marshal, M. P., Dietz, L. J., Friedman, M. S., Stall, R., Smith, H. A., McGinley, J., ... \& Brent, D. A. (2011). Suicidality and depression disparities between sexual minority and heterosexual youth: a meta-analytic review. Journal of Adolescent Health, 49(2), 115-123.

McGrath, R. E. (2005). Conceptual complexity and construct validity. Journal of personality assessment, 85(2), 112-124.

Meade, A. W., \& Wright, N. A. (2012). Solving the measurement invariance anchor item problem in item response theory. Journal of Applied Psychology, 97(5), 1016.

Méndez, C. \& Rondón, M. (2012). Introducción al análisis factorial. Revista Colombiana de Psiquiatría, 41(1).

Millham, J., San Miguel, C. L., \& Kellogg, R. (1976). A factor-analytic conceptualization of attitudes toward male and female homosexuals. Journal of Homosexuality, 2(1), 3-10.

Minton, H. L. (2002). Departing from deviance: A history of homosexual rights and emancipatory science in America. University of Chicago Press.

Moral, J., Valle, A., \& Martínez., E. (2013). Evaluación del rechazo hacia la homosexualidad en estudiantes de medicina y psicología con base en tres escalas conceptualmente afines. Psicología desde el Caribe, 30(3), 526550.

Moreno, A., Herazo, E., Oviedo, H., \& Campo-Arias, A. (2015). Measuring Homonegativity: Psychometric Analysis of Herek's Attitudes Toward Lesbians and Gay Men Scale (ATLG) in Colombia, South America. Journal of homosexuality, 62(7), 924-935.
Morrison, M. A., \& Morrison, T. G. (2011). Sexual Orientation Bias Toward Gay Men and Lesbian Women: Modern Homonegative Attitudes and Their Association With Discriminatory Behavioral Intentions1. Journal of Applied Social Psychology, 41(11), 2573-2599.

Morrison, M.A. \& Morrison, T.G. (2002). Development and validation of a scale measuring modern prejudice toward gay men and lesbian women. Journal of Homosexuality, 43(2), 15-37.

Movimiento de Integración y Liberación Homosexual \& Fundación Progresa (2012). Encuesta sobre discriminación de las minorías sexuales. Santiago, Chile. Recuperado de http://www.emol.com/documentos/archi vos/2011/10/06/20111006134740.pdf

Movimiento de Integración y Liberación Homosexual (2007). Informe Anual: derechos humanos de las minorías sexuales en Chile, hechos 2006. Santiago, Chile.

Movimiento de Integración y Liberación Homosexual (2008). VI Informe Anual: derechos humanos minorías sexuales chilenas, hechos 2007. Santiago, Chile.

Movimiento de Integración y Liberación Homosexual (2010). VIII Informe Anual: derechos humanos de la diversidad sexual chilena, hechos 2009. Santiago, Chile.

Movimiento de Integración y Liberación Homosexual (2012). X Informe Anual de Derechos Humanos de la Diversidad Sexual en Chile, Hechos 2011. Santiago, Chile. Recuperado de http://www.movilh.cl/documentacion/inf ormeddhh-2011/Informe-ddhh-MovilhChile-2011.pdf

Movimiento de Integración y Liberación Homosexual (2013). XI Informe Anual de Derechos Humanos de la Diversidad Sexual en Chile, Hechos 2012. Historia anual de las minorías sexuales chilenas. Santiago, Chile. Recuperado de http://www.movilh.cl/documentacion/XI 
Informe_de_DHH_Movilh_Hechos_20 12.pdf

Muñiz, J., Elosua, P., \& Hambleton, R. K. (2013). Directrices para la traducción y adaptación de los tests: segunda edición. Psicothema, 25(2), 151-157.

Muthén, B., \& Kaplan, D. (1992). A comparison of some methodologies for the factor analysis of non-normal Likert variables: A note on the size of the model. British Journal of Mathematical and Statistical Psychology, 45(1), 1930.

Nierman, A. J., Thompson, S. C., Bryan, A., \& Mahaffey, A. L. (2007). Gender role beliefs and attitudes toward lesbians and gay men in Chile and the US. Sex Roles, 57(1-2), 61-67.

O'Donohue, W., \& Caselles, C. E. (1993). Homophobia: Conceptual, definitional, and value issues. Journal of Psychopathol- ogy and Behavioral Assessment, 15, 177- 195.

Parrot, D., Adams, H. \& Zeichner, A. (2002). Homophobia: personality and attitudinal correlates. Personality and Individual Differences, 32(7), 1269-1278.

Plasek, W. J. \& Allard, J. (1984). Misconceptions of homophobia. En J. P. De Cecco (Ed.), Homophobia: An overview (pp. 23-37). New York, NY: Haworth Press.

Plummer, D. (1999). One of the boys: Masculinity, homophobia and modern manhood. Binghamton, NY: Haworth Press.

Reiter, L. (1991). Developmental origins of anti-homosexual prejudice in heterosexual men and women. Clinical Social Work Journal, 19, 163-175.

Robins, R. W., Hendin, H. M., \& Trzesniewski, K. H. (2001). Measuring global self-esteem: Construct validation of a single-item measure and the Rosenberg Self-Esteem Scale. Personality and social psychology bulletin, 27(2), 151-161.

Robles, R., \& Páez, F. (2003). Estudio sobre la traducción al español y las propiedades psicométricas de las escalas de afecto positivo y negativo (PANAS). Salud mental, 26(1), 69-75.

Roderick, T., McCammon, S. L., Long, T. E., \& Allred, L. J. (1998). Behavioral aspects of homonegativity. Journal of Homosexuality, 36(1), 79-88.

Romero, D. H., Morera, O. F., \& Wiebe, J. S. (2015). Assessing the Gender Invariance of the Modern Homonegativity Scale. Journal of Homosexuality, 62(11), 1539-1559. http://doi.org/10.1080/00918369.2015.1 073034

Satcher, J., \& Leggett, M. (2006). Homonegativity among Alabama Counselors. Alabama Counseling Association Journal, 32(2), 1-11.

Satcher, J., \& Leggett, M. (2007). Homonegativity among professional school counselors: An exploratory study. Professional School Counseling, 11(1), 10-16.

Schmitt, N., \& Kuljanin, G. (2008). Measurement invariance: Review of practice and implications. Human Resource Management Review, 18(4), 210-222.

Shackelford \& Besser (2007). Predicting Attitudes toward Homosexuality: Insights from Personality Psychology. Individual Differences Research, 5(2), 106-114.

Shields, S., Harriman, R. (1984). Fear of male homosexuality: cardiac responses of low and high homonegative males. Journal of Homosexuality, 10(1/2), 5367.

Smith, K. T. (1971) .Homophobia: A tentative personality profile. Psychological Reports, 29, 1091-1094

Thissen, D., Steinberg, L., \& Gerrard, M. (1986). Beyond group-mean differences: The concept of item bias. Psychological Bulletin, 99(1), 118.

Thompson, E. (2007). Development and validation of an internationally reliable short-form of the positive and negative affect schedule (Panas). Journal of cross-cultural psychology, 38(2), 227 242. 
Timmerman, M. E., \& Lorenzo-Seva, U. (2011). Dimensionality assessment of ordered polytomous items with parallel analysis. Psychological Methods, 16(2), 209.

Tucker, E. (2010). Towards a More Rigorous Scientific Approach to Social Measurement: Considering a Grounded Indicator Approach. The SAGE Handbook of Measurement, 313.

VIVOPOSITIVO \& ASOSIDA (2012). Índice compuesto de estigma y discriminación hacia hombres homosexuales, otros HSH y mujeres transgénero en Chile (ICED): sintesis de estudio. Santiago, Chile.

Watson, D., Clark, L. A., \& Tellegen, A. (1988). Development and validation of brief measures of positive and negative affect: the PANAS scales. Journal of personality and social psychology, 54(6), 1063.

Weinberg, G. H. (1972). Society and the healthy homosexual. Macmillan.

Whitley Jr, B. E. (2001). Gender-role variables and attitudes toward homosexuality. Sex Roles, 45(11-12), 691-721.

World Health Organization. (2010). Psychological and behavioural disorders associated with sexual development and orientation. International Statistical Classification of Diseases and Related Health Problems 10th Revision (ICD-10) Version for 2010. Recuperado de http://apps.who.int/classifications/icd10/ browse/2010/en\#/F66.1

World Health Organization. (2011). Stop discrimination against homosexual men and women. World Health Organization: Regional Office for Europe. Recuperado de http://www.euro.who.int/en/healthtopics/communicablediseases/hivaids/news/news/2011/5/sto p-discrimination-against-homosexualmen-and- women

Wrench, J. (2005). Development and Validity Testing of the Homonegativity
Short Form. Journal of Intercultural Communication Research, 34(3), 152165.

Wrench, J. S. (2001). Intercultural communication: Power in context.

Wu, A. D., Li, Z., \& Zumbo, B. D. (2007). Decoding the meaning of factorial invariance and updating the practice of multi-group confirmatory factor analysis: A demonstration with TIMSS data. Practical Assessment, Research \& Evaluation, 12, 1-26.

Ximénez, M. C., \& García, A. G. (2005). Comparación de los métodos de estimación de máxima verosimilitud y mínimos cuadrados no ponderados en el análisis factorial confirmatorio mediante simulación Monte Carlo. Psicothema, 17(3), 528-535.

Yang, A. S. (1997). The polls-trends. Attitudes toward homosexuality. Public Opinion Quarterly 61, 477-507. 